\title{
Self-Avoiding Walk in 5 or More Dimensions *
}

\author{
David Brydges ${ }^{1}$ and Thomas Spencer ${ }^{2}$ \\ 1 Department of Mathematics, University of Virginia, Charlottesville, VA 22903, USA \\ 2 Courant Institute of Mathematical Sciences, 251 Mercer Street, New York, NY 10012, USA
}

\begin{abstract}
Using an expansion based on the renormalization group philosophy we prove that for a $T$ step weakly self-avoiding random walk in five or more dimensions the variance of the endpoint is of order $T$ and the scaling limit is gaussian, as $T \rightarrow \infty$.
\end{abstract}

\section{Introduction and Results}

We consider walks $\omega(s)$ in $\mathbb{Z}^{d}$ which start at the origin and consist of $|\omega|=T$ nearest neighbor steps. If each such walk $\omega$ is assigned a weight proportional to

$$
P_{T}(\omega) \equiv \prod_{0<s<t \leqq T}(1-\lambda \delta(\omega(s)-\omega(t))), \quad 0<\lambda \leqq 1,
$$

we say that the walk is weakly self-avoiding or self-repelling. Here $s, t$ denote nonnegative integers and $\delta(j)=1$ if $j=0$ and $\delta(j)=0$ otherwise. When $\lambda=1$ only walks which strictly self-avoid are counted. Now let us define an expectation of a functional $F$ on paths $\omega,|\omega|=T$ by

$$
\langle F(\cdot)\rangle_{T}(\lambda) \equiv \frac{\sum_{|\omega|=T} F(\omega) P_{T}(\omega)}{\sum_{|\omega|=T} P_{T}(\omega)} .
$$

A natural quantity to study is the mean square displacement of $\omega(T)$ defined by

$$
R^{2}(T) \equiv\left\langle\omega^{2}(T)\right\rangle_{T}(\lambda) .
$$

In the physics literature $R^{2}(T)$ is expressed in terms of a critical exponent $v$ via the relation $R^{2}(T) \cong C(\lambda) T^{2 v}$ for large $T$. On the basis of renormalization group

Dedicated to the memory of Kurt Symanzik whose profound contributions have guided and inspired us

* Work partially supported by N.S.F. Grant DMR 81-00417

$\star \star \quad$ A. P. Sloan Foundation Fellow. Work partially supported by N.S.F. Grant MCS 82-02115 
considerations $v$ is supposed to be independent of $\lambda$ for $0<\lambda \leqq 1$ and $v \cong 0.75,0.58$ in dimensions 2 and 3 respectively [1]. In four or more dimensions $v$ is expected to be $\frac{1}{2}$ with logarithmic corrections in four dimensions [1].

On a mathematical level almost nothing has been proved about $v$, except when $d=1$ where $v=1$. For example it would be very interesting to obtain a lower bound on $v$ such as $v \geqq \frac{1}{2}$ or an upper bound $v \leqq 1-\varepsilon, \varepsilon>0$.

In this paper we examine the case $d \geqq 5$ for small positive values of $\lambda$. We prove the following theorem.

Theorem. For $d \geqq 5$, there is a $\lambda_{0}>0$ such that for each $\lambda, 0 \leqq \lambda \leqq \lambda_{0}$, there is $a$ positive constant $\bar{D}$ and

$$
\left\langle\omega^{2}(T)\right\rangle_{T}(\lambda)=D T\left(1+O\left(T^{-1 / 16}\right)\right) .
$$

Furthermore the scaling limit of the endpoint is gaussian, i.e.

$$
\lim _{s \rightarrow \infty}\left\langle e^{i k \omega(s t) / \sqrt{s}}\right\rangle_{s t}(\lambda)=e^{-D t k^{2} / 2}
$$

uniformly on compact sets of $k$ and $t$.

Dobrushin has informally announced results similar to ours but no proof has yet appeared.

A careful analysis of our proof shows that the coefficient of $\delta(\omega(s)-\omega(t))$ may be set equal to 1 for $|s-t| \geqq M$ large. The smallness in $\lambda$ is only needed for $|s-t| \leqq M$.

The role of dimension can heuristically be seen from the fact that two independent random walks intersect only finitely often with probability one in dimension $d \geqq 5$.

Our result exemplifies the validity of mean field theory in high dimensions. Another such example are the remarkable results of Aizenman [2] and Fröhlich [3] on the gaussian nature of $\lambda \phi^{4}$ field models in five or more dimensions. [Formally, self avoiding walk may be regarded as the $N \rightarrow 0$ limit of $\lambda \phi^{4}$ theories, where $N$ denotes the number of components of the field $\phi$.] Another related result is the work of Lawler [4] who proved that the scaling limit of a loop reased random walk is Brownian motion when $d \geqq 5$.

The proof of Theorem 1 is based on an expansion in $\lambda$ which uses the renormalization group philosophy. An outline of the proof appears in Sect. 2. We believe that our techniques will be useful for analysing a number of other problems such as random walk in a random environment. We refer the reader to [5] for other mathematical results which incorporate the renormalization group philosophy. We also recommend the viewpoint and results in [6], e.g. Sects. 1-4.

\subsection{Outline of Proof}

Let us consider walks which remember to self repel only for a time span $\tau$, thus we define $V_{s t}=\delta(\omega(s)-\omega(t))$, and

$$
C_{\tau}(x, T) \equiv(2 d)^{-T} \sum_{\substack{\omega,|\omega|=T \\ \omega(T)=x}} \prod_{\substack{0 \leq s<t \leq T \\|\bar{s}-t| \leqq \tau}}\left(1-\lambda V_{s t}\right) .
$$


We shall use the same letter $C$ with different arguments to denote its Fourier transform,

$$
C_{\tau}(k, T) \equiv \sum_{x} C_{\tau}(x, T) e^{i k \cdot x}
$$

and its Fourier-Laplace transform,

$$
C_{\tau}(k, z) \equiv \sum_{T=0}^{\infty} C_{\tau}(k, T) z^{T}
$$

Here $k$ lies in the torus dual to $\mathbb{Z}^{d}$. We are interested in the probability distribution of $\omega(T)$, the position after $T$ steps, and in particular its variance given by

$$
\left\langle\omega^{2}(T)\right\rangle_{\tau} \equiv \sum_{x} C_{\tau}(x, T) x^{2} / \sum_{x} C_{\tau}(x, T)
$$

The theory of generalized Markov processes shows that for some non-zero constant $D_{\tau}$

$$
\left\langle\omega^{2}(T)\right\rangle_{\tau}=D_{\tau}(T+o(T)) \quad \text { as } \quad T \rightarrow \infty
$$

[A proof of this, for $\lambda$ small, is also contained in this paper.] $D_{\tau}$ is called the diffusion coefficient and our major task is to obtain bounds on $D_{\tau}$ which are uniform in $\tau$ as $\tau \rightarrow \infty$ when the dimension $d \geqq 5$. We do it by setting $\tau \equiv T_{l} \equiv 2^{\ell^{\alpha}}-1$ and devising an inductive scheme of the form "true for $\ell \leqq m-1$ " $\Rightarrow$ "true for $\ell \leqq m ", m=1,2, \ldots, \ell \in \mathbb{R}^{+}$. We will write $C_{\ell}$ for $C_{T_{\ell}}$ etc.

If $\lambda=0$ or $\tau=0$, then the product in (2.1) is one, and using the independence of nearest neighbor steps it is easy to show that

$$
C_{0}(k, z)=(1-z D(k))^{-1} \text {, }
$$

where

$$
D(k)=\frac{\sum_{i=1}^{d} \cos k_{i}}{d} .
$$

Let $r_{\ell}(k)$ denote the radius of convergence of (2.3). Note that $r_{\ell}(0)$ is increasing in $\ell$ because $(1-\lambda V) \leqq 1$ and $r_{\ell}(k) \geqq r_{\ell}(0) \geqq 1$. For $\ell=0, r_{0}(k)=\left|D(k)^{-1}\right|$.

Our strategy is first to show that $C_{\ell}(k, z)$ is close to $C_{0}(k, z)$ in dimension $d \geqq 5$. To make this idea precise let $\Pi_{\ell}(k, z)$ and $F_{\ell}(k, z)$ be implicitly defined by

$$
C_{\ell}(k, z)=\left(1-z D(k)-\Pi_{\ell}(k, z)\right)^{-1}=F_{\ell}(k, z)^{-1} .
$$

We shall prove that $\Pi_{\ell}$ is analytic and for $|u| \leqq 2$,

$$
\left|\partial_{k}^{u} \Pi_{\ell}(k, z)\right|,\left|\frac{\partial}{\partial z} \Pi_{\ell}(k, z)\right| \leqq K \lambda,
$$

for

$$
z \in \mathscr{D}_{\ell} \equiv\left\{z:|z| \leqq r_{\ell}(0)+\frac{1}{16} T_{\ell}^{-1} \ln T_{\ell}\right\} .
$$

Here $K$ is a constant independent of $\ell$. 
We now explain how these estimates imply that the diffusion constant $D_{\ell}$ obtained using

$$
\left.\frac{-\Delta_{k} C_{\ell}(k, T)}{C_{\ell}(k, T)}\right|_{k=0}=D_{\ell} T(1+o(T))
$$

is uniformly founded. By (2.6) we see that $C_{\ell}(k, z)$ is bounded and meromorphic in $\mathscr{D}_{\ell}$ and has a unique real pole $r_{\ell}(k)$ for small values of $k$, which varies differentiably in $k$. See Sect. 6. By the Cauchy integral formula and (2.3),

$$
\begin{aligned}
r_{\ell}(0)^{T+1} C_{\ell}(k, T) & =\frac{1}{2 \pi i} \oint C_{\ell}(k, z)\left(\frac{r_{\ell}(0)}{z}\right)^{T+1} d z \\
& =-\left(\frac{\partial F_{\ell}}{\partial z}\right)^{-1}\left(\frac{r_{\ell}(0)}{r_{\ell}(k)}\right)^{T+1}+\frac{1}{2 \pi i} \oint_{\partial \mathscr{\partial}_{\ell}} C_{\ell}(k, z)\left(\frac{r_{\ell}(0)}{z}\right)^{T+1} d z \\
& \simeq\left(\frac{r_{\ell}(0)}{r_{\ell}(k)}\right)^{(T+1)} \simeq e^{-k^{2} T D_{\ell} / 2}
\end{aligned}
$$

Here we have deformed a small circle about the origin to $\partial \mathscr{D}_{\ell}$ to pick up the contribution from the pole at $r_{\ell}(k)$ plus an error term which is shown to be small for large $T \geqq T_{\ell}$ in Sect. 7. Roughly speaking the error term is small because

$$
\left|\frac{r_{\ell}(0)}{z}\right|^{T}=\left[r_{\ell}(0) /\left(r_{\ell}(0)+\frac{1}{16} \frac{\ln T_{\ell}}{T_{\ell}}\right)\right]^{T} \approx e^{-\frac{1}{16} \frac{T}{T_{\ell}} \ln T_{\ell}} .
$$

Now the diffusion constant is easily calculated in terms of the second derivative of $r_{\ell}(k)$ at $k=0$ (by symmetry $r_{\ell}^{\prime}(0)=0$ ). Uniform bounds on $r_{\ell}^{\prime \prime}(0)$ are obtained using the implicit function theorem and (2.6).

In order to obtain bounds of the form (2.6) on $\Pi_{\ell}(k, z)$ we first develop a formula for $\Pi_{\ell}$ in terms of a convergent series. A detailed derivation of this formula in presented in Sect. 4. Section 5 is the core of this paper. It is there where our induction hypothesis is formulated and a bound on $\Pi_{\ell}$ and $\delta \Pi_{\ell}=\Pi_{\ell}-\Pi_{\ell-1}$ is obtained in terms of $L_{p}$ norms of $C_{\ell-1}(x, T)$.

In the remainder of this section we shall sketch a formula for $\Pi_{\ell}$ and some of the ideas of Sect. 5. Let

$$
U_{s t}=-\lambda V_{s t} ; \tau=T_{\ell} .
$$

We begin by expanding the "interaction"

$$
\prod_{|s-t| \leqq \tau}\left(1+U_{s t}\right)=\sum_{\Gamma} \prod_{s t \in \Gamma} U_{s t},
$$

where $\Gamma$ ranges over all collections of "lines" or pairs $s, t$ such that

$$
0 \leqq s<t \leqq T \text { and }|t-s| \leqq \tau .
$$

Any such collection of lines is called a graph. A time $a, 0<a \leqq T$, is said to be a Markov time for $\Gamma$ if there is no line $s t \in \Gamma$ such that $s<a<t$. Thus there is no interaction (or line) across $a$. A graph $\Gamma$ on $[0, T]$ is said to be primitive if it has no Markov points in $(0, T)$ and 0 belongs to some line of $\Gamma$. Every graph decomposes uniquely into primitive subgraphs over the appropriate intervals and it is not 
difficult to prove that this leads to the formula

$$
\Pi_{\ell}(k, z)=\sum_{T} z^{T} \Pi_{\ell}(k, T),
$$

where

$$
\Pi_{\ell}(k, T)=\sum_{|\omega|=T} \sum_{T} \prod_{\substack{\text { primitive } \\ \text { on }[0, T]}} \prod_{s t \in \Gamma} U_{s t} e^{i k \omega(T)}
$$

(see Sect. 4). There are too many graphs in (2.10) in the sense that if absolute values are taken inside (2.11), (2.10) will diverge. For this reason we resum our family of primitive graphs. To each primitive graph $\Gamma$ we associate a (unique) minimal subgraph $S(\Gamma) \subset \Gamma$ which is a primitive subgraph of $\Gamma$ with as few lines as possible (see Fig. 1 below). We call such a graph $S(\Gamma)$ a lace. The precise definition of $S(\Gamma)$ is given in Sect. 4.
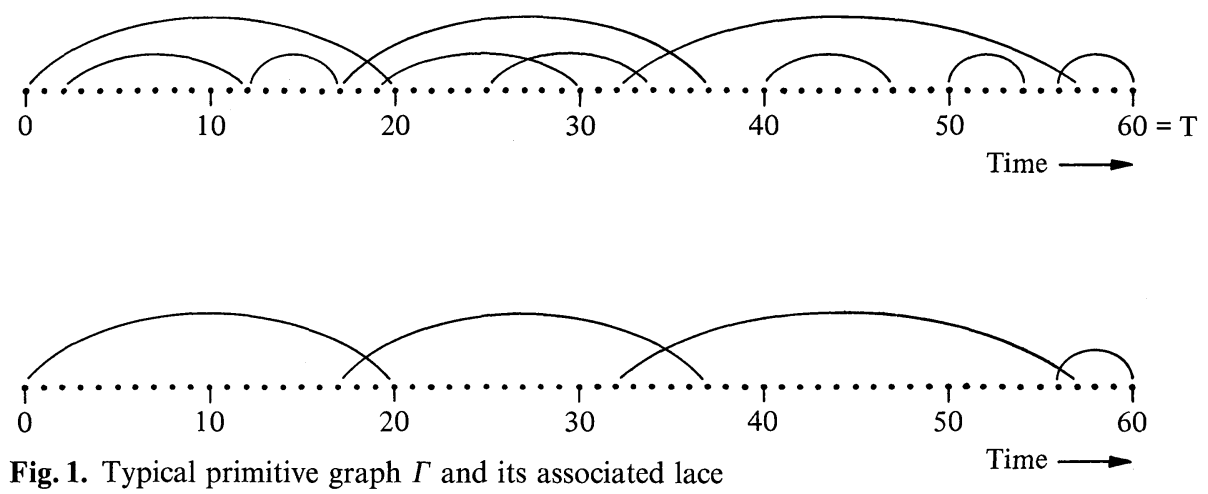

Fig. 1. Typical primitive graph $\Gamma$ and its associated lace

We now sum over all primitive graphs $\Gamma$ with $S(\Gamma)=L$ held fixed and then sum over $L$. The sum over $\Gamma$ with $S(\Gamma)=L$ reconstructs part of the interaction, cf. (2.9), indeed

$$
\sum_{\Gamma: S(\Gamma)=L} \prod U_{s t}=\prod_{s t \in \mathscr{G}_{\tau}(L)}\left(1+U_{s t}\right) \prod_{s t \in L} U_{s t} .
$$

Here $\mathscr{C}_{\tau}(L)$ is defined in Sect. 4 to be the set of lines compatible with $L$. Our desired expression for $\Pi_{\ell}$ is

$$
\Pi_{\ell}(k, T)=\sum_{L} \sum_{\substack{\omega \\|\omega|=T}} \prod_{s t \in \mathscr{C}_{\tau}(L)}\left(1+U_{s t}\right) \prod_{s t \in L} U_{s t} e^{i k \cdot \omega(T)},
$$

where $\sum_{L}$ is the sum over all lacings on $[0, T]$.

Let $\Pi^{N}$ denote the contribution to the sum in (2.12) arising from all $N$ lacings, i.e. lacings with precisely $N$ lines. Then we have

$$
\left|\frac{\partial}{\partial z} \Pi_{\ell}(k, z)\right| \leqq \sum_{N}\left|\frac{\partial}{\partial z} \Pi_{\ell}^{N}(k, z)\right| .
$$


In Sect. 5 we prove that the right side is convergent for $z \in \mathscr{D}_{\ell}$ uniformly in $\ell$ and each term is bounded in terms of the $L_{p}$ norms of $r_{\ell-1}(0)^{T} C_{\ell-1}(x, T), p \geqq 2$, which by inductive assumption is less than $\left\|K \exp \left(-D_{\ell-1} k^{2} T\right)\right\|$, so that e.g. in $x$ space,

$$
r_{\ell-1}(0)^{T}\left\|C_{\ell-1}(\cdot, T)\right\|_{L^{\infty}} \leqq \text { const } T^{-d / 2} .
$$

We illustrate this method by bounding

$$
\left|\frac{\partial}{\partial \mathrm{z}} \Pi_{t}^{1}\right| \text {. }
$$

When $N=1, L=\{(0, T)\}$, and $\mathscr{C}(L) \equiv \mathscr{C}_{T}=$ set of all pairs $s t, 0 \leqq s<t \leqq T$ such that $|s-t| \leqq T_{\ell}$ and $|s-t|<T$. Thus

$$
\left|\Pi_{\ell}^{1}(k, T)\right|=\lambda\left|\sum_{|\omega|=T} \prod_{s t \in \mathscr{C}_{T}}\left(1-\lambda V_{s t}\right) V_{0 T} e^{i \omega(T) \cdot k}\right| .
$$

The factor $V_{0 T}$ above ensures that $\omega(T)=0$ so that

$$
\left|\Pi_{\ell}^{1}(k, T)\right|=\lambda \sum_{\substack{|\omega|=T \\ \omega(T)=0}} \Pi\left(1-\lambda V_{s t}\right) \leqq \frac{\lambda}{1-\lambda} C_{\ell-1}(x=0, T) .
$$

In the last inequality we have used (2.1) and the fact that $0 \leqq(1-\lambda V) \leqq 1$. By inductive assumption [see (2.8) and Sect. 5 for details]

$$
\begin{aligned}
r_{\ell-1}(0)^{T} C_{\ell-1}(x=0, T)= & r_{\ell-1}(0)^{T} \int C_{\ell-1}(k, T) d k /(2 \pi)^{d} \\
& <K T^{-d / 2} .
\end{aligned}
$$

When $T>T_{\ell}, \Pi^{1}=0$, thus, by $(2.10)$,

$$
\begin{aligned}
\left|\frac{\partial \Pi^{1}}{\partial z}\right| & \leqq \lambda \sum_{1 \leqq T \leqq T_{\ell}} T|z|^{(T-1)} \Pi^{1}(k, T) \\
& \leqq \lambda K \sum_{1 \leqq T \leqq T_{\ell}} T^{(1-d / 2)}\left|\frac{z}{r_{\ell-1}(0)}\right|^{T} .
\end{aligned}
$$

Now for $d \geqq 5$ the right side converges uniformly in $T_{\ell}$ and $z \in \mathscr{D}_{\ell}$, so we have obtained part of (2.6) inductively ${ }^{1}$. In Sect. 7 it is proved, along the lines of our discussion of (2.8), that the bound on $\Pi,(2.6)$, allows us to advance the inductive assumptions on $C$.

\section{Notation and Conventions}

In this paper we use $K$ to denote a constant independent of all parameters, except the dimension $d$. In particular $K$ is independent of the induction step, labelled by $m=1,2, \ldots$. For constants which do not enjoy these properties we will indicate dependences as arguments, e.g. $K(\delta) . E$, as in error, is used to denote a function of $k, z, \lambda$ which is bounded in absolute value by some $K . K$ and $E$ are not necessarily

1 We are omitting an argument that $r_{\ell-1}(0) \simeq r_{\ell}(0)$, when $\ell$ is large or $\lambda$ small. In addition we have simplified the estimate of the sum over $T$ which in fact involves disection into subranges according to values of $T_{\ell^{\prime}}, \ell^{\prime} \leqq \ell$ 
the same in different locations including strings of inequalities for the same quantity. The rest of this section is a compendium of our notation.

For each $\ell \in \mathbb{R}$ we define a memory $T_{\ell}$ by

$$
T_{\ell} \equiv \max \left(2^{\ell \alpha}-1,0\right) \quad \text { with } \quad \alpha \equiv 9 / 8 .
$$

We also define $\ell(s)$ by $T_{\ell(s)}=s$. To each $\ell$ is associated a random walk, self-avoiding (with strength $\lambda$ ) with memory $T_{\ell}$,

$$
\begin{gathered}
C_{\ell}(x, T) \equiv \sum_{\omega: 0 \rightarrow x,|\omega|=T}(2 d)^{-T} \prod_{\substack{s t,|t-s|<T_{\ell} \\
0 \leqq s<t \leqq T_{\ell}}}\left(1-\lambda V_{s t}\right), \\
V_{s t} \equiv \delta(\omega(s)-\omega(t)) \equiv \begin{cases}1 & \text { if } \omega(s)=\omega(t), \\
0 & \text { else }\end{cases}
\end{gathered}
$$

$0 \leqq \lambda \leqq 1, T=1,2, \ldots$.

$$
C_{\ell}(k, T) \equiv \sum_{\omega:|\omega|=T}(2 d)^{-T} \prod_{\substack{s t,|t| s \mid \leq T_{\ell} \\ 0 \leqq s<t \leqq T}}\left(1-\lambda V_{s t}\right) e^{i k \omega(T)} .
$$

This is the fourier transform of $C_{t}(x, T)$,

$$
\begin{aligned}
\left\langle e^{i k \omega(T)}\right\rangle_{\ell, T} & \equiv C_{\ell}(k, T) / C_{\ell}(0, T), \\
C_{\ell}(k, z) & \equiv \sum_{T=0}^{\infty} z^{T} C_{\ell}(k, T) .
\end{aligned}
$$

By convention $C_{\ell}(k, T=0) \equiv 1$. The arguments are to be used to distinguish the different transforms being denoted by $C$ 's,

$$
F_{\ell}(k, z) \equiv\left[C_{\ell}(k, z)\right]^{-1} .
$$

$r_{\ell}$ is the radius of convergence of $C_{t}(k=0, z)$. It will be seen that $F_{t}(k=0, z)$ has a simple zero at $z=r_{\ell}$ and the trajectory in $\mathbb{C}$ of this zero as $k$ varies will be denoted by $r_{\ell}(k)$,

$$
D(k) \equiv \frac{1}{d} \sum_{i=1}^{d} \cos k_{i}
$$

$k_{i}, \ldots, k_{d}$ are the components at $k . \Pi_{\ell}(k, z)$ is defined implicitly by

$$
\begin{gathered}
C_{\ell}(k, z) \equiv\left[1-z D(k)-\Pi_{\ell}(k, z)\right]^{-1}, \\
\delta \Pi_{\ell}(k, z) \equiv \Pi_{\ell}(k, z)-\Pi_{\ell-1}(k, z), \\
\varepsilon_{\ell} \equiv \frac{1}{16} \ln \left[2+T_{\ell}\right] /\left[2+T_{\ell}\right], \\
d(u, v) \equiv \frac{d}{2}-\alpha\left(\frac{|u|}{2}+v\right)-\frac{9}{8} .
\end{gathered}
$$

$u$ is a multi-index which we use for $k$ derivatives denoted by $\partial_{k}^{u}$. Likewise $\partial_{z}^{v}$ denotes $z$ derivatives,

$$
\mathscr{D}_{\ell}(a) \equiv\left\{z \in \mathbb{C}:|z| \leqq r_{\ell}+a \varepsilon_{\ell}\right\} ; a>0 .
$$

Throughout this paper $d \geqq 5$ and $\lambda$ is assumed non-negative and small. 


\section{A Formula for $\Pi$}

The interaction of a path with itself over a time interval $[0, T]$ is given by

$$
P[0, T] \equiv \prod_{0 \leqq s<t, t-s \leqq \tau}\left(1+U_{s t}\right),
$$

where

$$
U_{s t} \equiv-\lambda V_{s t} \equiv-\lambda \delta(\omega(s)-\omega(t)) .
$$

$\tau\left(=T_{\ell}\right)$ is the memory over which the random walk $\omega$ self-avoids. Following the outline given in Sect. 2 we will develop an expansion for $P$ and deduce from it a formula for $\Pi$ which appears in Theorem 4.1 below.

We expand $P$ as a sum of all graphs as explained in Sect. 2. For each graph $\Gamma$ we let $a(\Gamma), 0<a(\Gamma) \leqq T$, denote the first Markovian time of $\Gamma$, as defined in Sect. 2. Then

$$
P[0, T]=P[1, T]+\sum_{a=1}^{T} K[0, a] P_{0}[a, T],
$$

where

$$
K[0, a]=\sum_{\substack{\Gamma \text { primitive } \\ \text { on }[0, a]}}, \prod_{s t \in \Gamma} U_{s t} .
$$

The $P[1, T]$ is there because we insist $a(\Gamma)>0$. It is the sum of all graphs with no bond joining 0 to some other time. The second equality follows by noticing that we can split $\Gamma$ into two disjoint subsets corresponding to bonds on $[0, a],[a, T]$, and sum over them independently.

The last equality implies

$$
\begin{aligned}
C_{\tau}(k, z) \equiv & \sum_{T=0}^{\infty}\left(\frac{z}{2 d}\right)^{T} \sum_{\omega,|\omega|=T} P[0, T] e^{i k \omega(T)} \\
= & 1+z D(k) C_{\tau}(k, z)+\sum_{T=1}^{\infty}\left(\frac{z}{2 d}\right)^{T} \sum_{a=1}^{T} \sum_{\omega,|\omega|=T} \\
& \cdot K[0, a] e^{i k \omega(a)} P[a, T] e^{i k(\omega(T)-\omega(a))} .
\end{aligned}
$$

(By convention $P[0,0]=1$.) We can factor the sum over $\omega$ across the time $a$ and continue with

$$
=1+z D(k) C_{\tau}(k, z)+\left\{\sum_{a=1}^{\infty}\left(\frac{z}{2 d}\right)^{a} \sum_{\omega,|\omega|=a} K[0, a] e^{i k \omega(a)}\right\} C_{\tau}(k, z) .
$$

On recalling that $\Pi_{\tau}$ is defined implicitly by

$$
C_{\tau}(k, z) \equiv\left[1-z D(k)-\Pi_{\tau}(k, z)\right]^{-1},
$$

it follows that

$$
\Pi_{\tau}(k, z)=\sum_{a=1}^{\infty}\left(\frac{z}{2 d}\right)^{a} \sum_{\omega,|\omega|=a} K[0, a] e^{i k \omega(a)} .
$$


The next step is to find a better expression for $\Pi$, which unlike the one above will be absolutely convergent, i.e. absolute values can be taken inside all sums including the sum over graphs inside $K[0,1]$ provided $\lambda$ is small. Given a graph $\Gamma$ on $[0, a]$ we define a subgraph $S(\Gamma) \equiv L$ consisting of bonds $s_{i} t_{i}, i=1,2, \ldots, N_{\Gamma}$ obtained inductively by

$$
\begin{gathered}
t_{1} \equiv \max \{t: 0 t \in \Gamma\}, \\
t_{j} \equiv \max \left\{t: s t \in \Gamma, s<t_{j-1}\right\} ; j \geqq 2, \\
N_{\Gamma} \equiv \text { first value of } j \text { for which } t_{j}=a .
\end{gathered}
$$

Having obtained $t_{j}, s_{j}$ is defined by

$$
s_{j} \equiv \min \left\{s: s t_{j} \in \Gamma\right\} .
$$

The set of all $L$ that are obtained by this process applied to $\Gamma$ as $\Gamma$ runs over all graphs which occur in $K[0, a]$, (primitive graphs), is denoted $\mathscr{L}_{\tau}[0, a]$. If $L \in \mathscr{L}_{\tau}[0, a]$ we call it a lace. If it has $N$ bonds it is called an $N$-lace. It is not difficult to see that $L=\left\{s_{i} t_{i}: i=1,2, \ldots, N\right\} \in \mathscr{L}_{\tau}[0, a]$ iff
a) $s_{1}=0, t_{N}=a,\left|t_{j}-s_{j}\right| \leqq \tau$ for $j=1,2, \ldots, N$,
b) for $j=2,3, \ldots, N-1 s_{j} t_{j}$ overlaps with$$
s_{j-1} t_{j-1} \text { and } s_{j+1} t_{j+1} \text {. No other overlaps occur. }
$$

We say that $s t$ overlaps with $s^{\prime} t^{\prime}$ iff the open intervals $(s, t),\left(s^{\prime}, t^{\prime}\right)$ intersect.

The discussion so far shows that

$$
K[0, a]=\sum_{L \in \mathscr{L}_{\tau}[0, a]}\left(\sum_{\Gamma, S(\Gamma)=L} \prod_{s t \in \Gamma / L} U_{s t}\right) \prod_{s t \in L} U_{s t} .
$$

In order to simplify the sum in round brackets we observe that $\Gamma$ satisfies $S(\Gamma)=L$ $=\left\{s_{i} t_{i}: i=1,2, \ldots\right\}$ iff $\Gamma / L$ is a subset of $\mathscr{C}_{\tau}(L)$ which, by definition, is all bonds $s t$ satisfying

a) $0 \leqq s<t \leqq a,|t-s| \leqq \tau$, st $\notin L$.

b) If $s=0, t<t_{1}$. If $s<t_{i}$ for some $i$, then $t \leqq t_{i+1}$.

c) If $t=t_{i}$, then $s>s_{i}$.

This assertion is an easy consequence of the definition of $L$. It follows, cf. (2.9), that

$$
\sum_{\Gamma, S(\Gamma)=L} \prod_{s t \in \Gamma / L} U_{s t}=\prod_{s t \in \mathscr{C}_{\tau}(L)}\left(1+U_{s t}\right) .
$$

When we collect up (4.1), (4.2), (4.4), (4.6) we obtain

\section{Theorem 4.1.}

$\Pi_{\tau}(k, z)=\sum_{T \geqq 1} \sum_{\omega:|\omega|=T}\left(\frac{z}{2 d}\right)^{T} e^{i k \omega(T)} \sum_{L \in \mathscr{L}_{\tau}[0, T]} \prod_{s t \in L}\left(-\lambda V_{s t}\right) \prod_{s t \in \mathscr{C}_{\tau}(L)}\left(1-\lambda V_{s t}\right)$,

where $V_{s t}=\delta(\omega(s)-\omega(t)), \mathscr{L}_{\tau}[0, T]$ is the set of laces defined in (4.3) (with $a=T$ ) and $\mathscr{C}_{\tau}(L)$ is the set of bonds compatible with $L$ defined in (4.5). 
We will use this theorem in Sect. 5 to get good bounds on $\Pi$. The following features of $\mathscr{C}_{\tau}(L)$ will be needed: if $L$ is an $N$-lace, $N>1$, on $[0, T]$, then its bonds $s_{i} t_{i}$ define a partition of $[0, T]$ into $2 N-1$ subintervals $I_{1}, \ldots, I_{2 N-1}$ with disjoint interiors, obtained by putting the times $s_{i}, i=1,2, \ldots, N, t_{j}, j=1,2, \ldots, N$ in order and letting $I_{k}, k=1,2, \ldots, 2 N-1$, be the intervals between (see Fig. 2). The properties (4.5) imply that

$$
s t \in \mathscr{C}_{\tau}(L) \text { if }|t-s| \leqq \tau \text { and } s, t \in I_{j} \text { for some } j .
$$

This means that the random walk $\omega$ restricted to any $I_{j}$ is still self-avoiding.

Fig. 2

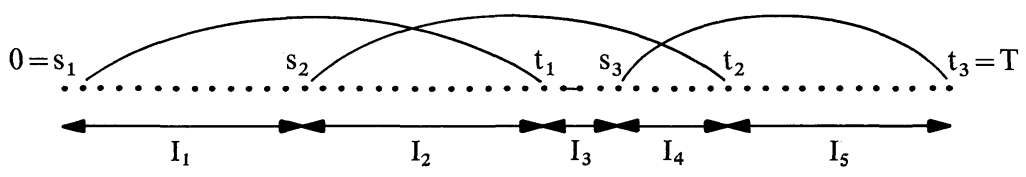

The other feature of $\mathscr{C}_{\tau}(L)$ which we will use in Sect. 5 is that if all the bonds $s t \in L$ satisfy $|t-s| \leqq \tau / 2$, then

$$
\mathscr{C}_{\tau^{\prime}}(L)=\mathscr{C}_{\tau}(L) \text { for } \tau^{\prime} \geqq \tau,
$$

because of condition (b) in (4.5).

\section{Inductive Assumptions on $\boldsymbol{C}$, Consequences for $\boldsymbol{\delta} \Pi$}

The induction is on $m=1,2, \ldots$. The inductive assumptions are: there exist $K^{(\mathrm{I} 1)}$, $K^{(I 2)}$ fixed throughout our induction such that

(I1) $0 \leqq r_{\ell}-r_{\ell-1} \leqq K^{(\mathrm{II} 1)} \sqrt{\lambda}\left(2+T_{\ell-1}\right)^{9 / 8-d / 2}$ for $\ell \leqq m-1$,

(I2) $\left\|\partial_{k}^{u} C_{\ell}(\cdot, T)\right\|_{p} \leqq K^{(\mathrm{I} 2)} r_{\ell}^{-T} T^{|u| / 2-d /(2 p)}$

$$
\text { for } \ell \leqq m-1, T \geqq T_{\ell+1}, T \geqq 1,|u| \leqq 2 .
$$

(I2) says that for large $T r_{\ell}^{T} \partial_{k}^{u} C(k, T) \cong \partial_{k}^{u} e^{-K k^{2} T}$ in $L_{p}$ norm. At the beginning of the induction $m=1$ so $\ell \leqq 0$. The definitions of $r_{\ell}, C_{\ell}$ for negative $\ell$ imply that $r_{\ell}=r_{0}$, $C_{\ell}=C_{0}$ so (I1) is true and (I2) holds by direct calculation on $C_{0}(k, T)=D(k)^{T}$, provided we take $K^{(\mathbf{I} 2)}$ sufficiently large.

In this section we will first prove a bound on $\delta \Pi_{\ell}=\Pi_{\ell}-\Pi_{\ell-1}$ in terms of $x$ space $L_{p}$ norms of $C_{\ell-1}$. This is Proposition 5.2 given below. $x$-space $L_{p}$ norms are convenient since we can use $x$-space bounds such as $1-\lambda V \leqq 1$ on them. They are converted to $k$-space $L_{p}$ bounds by the Hausdorff-Young inequality, and then the inductive assumptions are applied to obtain our main result for this section which is:

Proposition 5.1. (I1) and (I2) imply that if $\lambda$ is smaller than a universal constant, then

$$
\left|\partial_{k}^{u} \partial_{z}^{v} \delta \Pi_{\ell}(k, z)\right| \leqq K \lambda\left[2+T_{\ell-1}\right]^{-d(u, v)},
$$

for $\ell \leqq m,|u| \leqq 2$, and $z$ in the disc

$$
\left\{z:|z| \leqq r_{\ell-1}+2 \varepsilon_{\ell}\right\} .
$$


$\partial_{k}^{u} \partial_{z}^{v} \delta \Pi_{\ell}(k, z)$ is analytic on this disc and continuous in $k$. The constants $d(u, v), \varepsilon_{\ell}$ are given by

$$
\begin{gathered}
d(u, v) \equiv \frac{d}{2}-\alpha\left(\frac{|u|}{2}+v\right)-\frac{9}{8} \\
\varepsilon_{\ell} \equiv \frac{1}{16} \ln \left[2+T_{\ell}\right] /\left[2+T_{\ell}\right] .
\end{gathered}
$$

Notice that we have started our inductive step because in Proposition 5.1 $\ell \leqq m$ as opposed to the $m-1$ in the inductive assumptions. In Sect. 6 we will use Proposition 5.1 to advance (I1) to $\ell \leqq m$. In Sect. 7 we will use Proposition 5.1 and results from Sect. 6 to advance I2.

Estimates on $\delta \Pi$. To illustrate the basic strategy we will first obtain a weak version of the estimate we will ultimately need.

We start with the expression of Theorem 4.1 for $\Pi_{\ell}$ and estimate $\left|\Pi_{\ell}\right|$ by taking absolute values inside the sums over $T, L$, and $\omega$, and use

$$
|1-\lambda \delta(\omega(s)-\omega(t))| \leqq 1
$$

on all $s t \in \mathscr{C}_{\ell}(L)$. The result is

$$
\left|\Pi_{\ell}(k, z)\right| \leqq \sum_{T=1}^{\infty} \sum_{\omega:|\omega|=T}\left(\frac{|z|}{2 d}\right)^{T} \sum_{L \in \mathscr{L}_{\ell}[0, T]}, \quad \prod_{s t \in L} \lambda \delta(\omega(s)-\omega(t)) .
$$

The contribution of each $L$ can be expressed in terms of $C_{0}(x, z)$; consider the first three laces

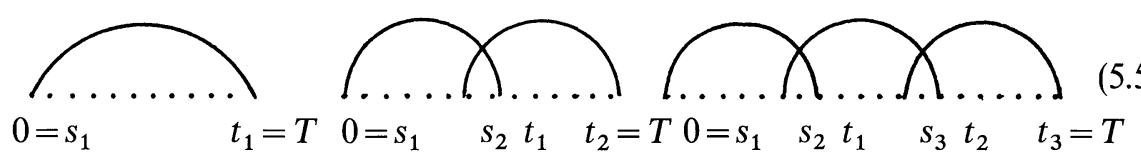

These diagrams, according to the product over st $\in$ Lin (5.4) correspond to the sum of random walks that intersect themselves as indicated in the figure below
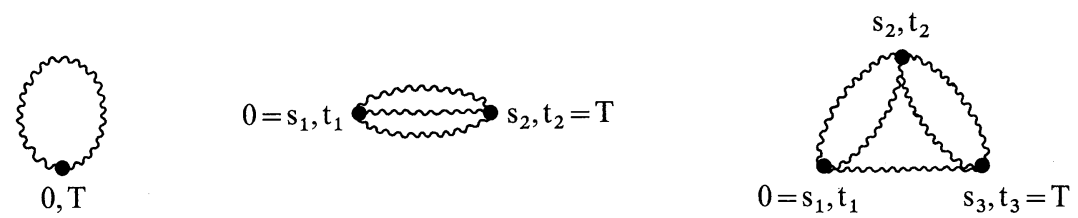

where the times opposite each intersection indicate the times at which the intersection takes place. With $L$ and positions of intersection held fixed, we can rewrite the sum over $\omega$ in (5.4) as separate sums over $\omega_{1}, \omega_{2}, \ldots$, where $\omega_{i}$ is the restriction of $\omega$ to the time interval $I_{i}$ in Fig. 2. We can also introduce times $\sigma_{i} \geqq 1$ for each $\omega_{i}$ :

$$
\sigma_{1}=s_{2}-s_{1}, \sigma_{2}=t_{1}-s_{2}, \sigma_{3}=s_{3}-t_{1}, \ldots,
$$


and replace the sum over $T$ by sums over $\sigma_{1}, \sigma_{2}, \ldots \leqq T_{\ell}$ separately. Define

$$
\tilde{C}_{0}(x, z)=\sum_{\sigma \leqq T_{\ell}} z^{\sigma} C_{0}(x, \sigma) .
$$

Now we can write down the result of doing the sums over $\sigma_{1}, \sigma_{2}, \ldots$, and positions of intersection. The contributions of the three diagrams $\left(5.5^{\prime}\right)$ to the righ-hand side of our upper bound on $\left|\Pi_{\ell}\right|$ are less than

$$
\lambda \widetilde{C}(0,|z|), \lambda^{2} \sum_{x} \tilde{C}_{0}^{3}(x,|z|), \lambda^{3} \sum_{x}\left(\left(\tilde{C}_{0}^{2} * \tilde{C}_{0}^{2}\right) \tilde{C}_{0}\right)(x,|z|),
$$

where $*$ denotes convolution. Let $\mathbf{M}$ denote the operation of multiplying by $\widetilde{C}_{0}$, i.e.

$$
\mathbf{M}: f(x) \rightarrow \widetilde{C}_{0}(x,|z|) f(x),
$$

and let $\mathbf{C}$ denote convolution by $\tilde{C}_{0}$ :

$$
\mathbf{C}: f(x) \rightarrow \sum_{y} \widetilde{C}_{0}(x-y) f(y) .
$$

Then these three contributions may be rewritten in the form

$$
\lambda \widetilde{C}_{0}(0,|z|), \lambda^{2}(\mathbf{C M} \tilde{C})(0,|z|), \lambda^{3}\left(\mathbf{C M C M} \tilde{C}_{0}\right)(0,|z|) .
$$

This pattern continues; the next diagrams are
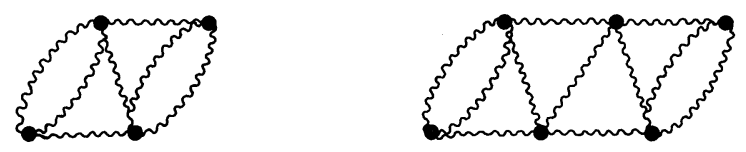

which correspond to

$$
\lambda^{4}\left((\mathbf{C M})^{3} \tilde{C}_{0}\right)(0,|z|), \lambda^{5}\left((\mathbf{C M})^{4} \tilde{C}_{0}\right)(0,|z|), \ldots,
$$

and an easy induction shows that this pattern persists so that the right-hand side of (5.4) equals

$$
\sum_{i=0}^{\infty}\left((\mathbf{C M})^{i} \widetilde{C}_{0}\right)(0,|z|) \lambda^{i+1}
$$

We now introduce a bound for each term in this sum whose content is that each term may be bounded by a product of $L^{2}$ norms of $\tilde{C}_{0}$ with one factor of $\tilde{C}_{0}$ omitted and instead bounded by an $L_{\infty}$ norm. Let, for $j=1, \ldots, i, \mathbf{C}_{2 j}$ be convolution by a function $f_{2 j}$ and $\mathbf{M}_{2 j}$ be multiplication by $f_{2 j-1}$, then we claim that

$$
\left\|\prod_{j=1}^{i}\left(\mathbf{C}_{2 j} \mathbf{M}_{2 j}\right) f_{0}\right\|_{\infty} \leqq\left(\prod_{j=1, j \neq k}^{2 i}\left\|f_{j}\right\|_{2}\right)\left\|f_{k}\right\|_{\infty},
$$

for any $k=1, \ldots, 2 i$.

Proof. We use Young and Hölder's inequalities in the forms

$$
\begin{array}{cc}
\mathbf{M}_{f}: L_{2} \rightarrow L_{1}, & \left\|\mathbf{M}_{f}\right\| \leqq\|f\|_{2}, \\
\mathbf{C}_{g}: L_{1} \rightarrow L_{2}, & \left\|\mathbf{C}_{g}\right\| \leqq\|g\|_{2},
\end{array}
$$


so that

$$
\left\|\mathbf{C}_{g} \mathbf{M}_{f}\right\|_{L_{2} \rightarrow L_{2}} \leqq\|f\|_{2}\|g\|_{2} .
$$

Also using $\mathbf{M}_{f}: L_{\infty} \rightarrow L_{2}, \mathbf{C}_{g}: L_{2} \rightarrow L_{\infty}$, we obtain

$$
\left\|\mathbf{C}_{g} \mathbf{M}_{f}\right\|_{L_{\infty} \rightarrow L_{\infty}} \leqq\|f\|_{2}\|g\|_{2} \text {. }
$$

Finally, using $\mathbf{M}_{f}: L_{2} \rightarrow L_{2}$ or $L_{1}$ and $\mathbf{C}_{g}: L_{2}$ or $L_{1} \rightarrow L_{\infty}$, we obtain

$$
\left\|\mathbf{C}_{g} \mathbf{M}_{f}\right\|_{L_{2} \rightarrow L_{\infty}} \leqq\left\{\begin{array}{l}
\|f\|_{\infty}\|g\|_{2} \\
\|f\|_{2}\|g\|_{\infty}
\end{array} .\right.
$$

The claim is a combination of these bounds.

We return to (5.7) and apply this bound (5.8) to obtain

$$
\left|\Pi_{m}(k, z)\right| \leqq \sum_{i=1}^{\infty} \prod_{j=1}^{2 i-1}\left(\left\|\widetilde{C}_{0}(\cdot,|z|)\right\|_{*}\right) \lambda^{i},
$$

where $*=2$ or $\infty$, with one \|\|$_{\infty}$.

Now this bound is insufficient for our needs but without destroying the structure of our argument it can easily be improved: in (5.4) it is not necessary to "throw away" all factors $1-\lambda V_{s t}$ by the upper bound (5.3). Instead we can remove only those factors $1-\lambda V_{\text {st }}$ which couple walks $\omega_{i}, \omega_{j}, i \neq j$ in our argument above. These are the bonds which couple the parts of $\omega$ in a way which is inconsistent with the factorization of the sum over $\omega$ (lace $L$ fixed) into independent sums over subwalks $\omega_{1}, \omega_{2}, \ldots$. With these bonds removed by a bound, the previous argument may be repeated yielding a bound in terms of $L_{2}, L_{\infty}$ norms of

$$
\sum_{s \leqq T_{\ell}}|z|^{s} C_{\ell}(x, s) .
$$

See (4.8) and the remark after it. Since $s \leqq T_{\ell}$ this equals

$$
\tilde{C}_{\ell}(x,|z|) \equiv \sum_{s \leqq T_{\ell}}|z|^{s} C_{\ell(s)}(x, s),
$$

where $\ell(s)$ is defined by $T_{\ell(s)}=s$, so that

$$
\left|\Pi_{\ell}(k, z)\right| \leqq \frac{1}{1-\lambda} \sum_{i=1}^{\infty} \prod_{i=1}^{2 i-1}\left(\left\|\tilde{C}_{\ell}(\cdot,|z|)\right\|_{*}\right) \lambda^{i}
$$

The $(1-\lambda)^{-1}$ restores the $0 T$ bond in the first diagram in (5.5).

Next we consider $\delta \Pi_{\ell}: \delta \Pi_{\ell} \equiv \Pi_{\ell}-\Pi_{\ell-1}$. The expansion in Theorem 4.2 is applied to each $\Pi$ on the right-hand side and we see that laces $L$ in which every bond $s t \in L$ is short, i.e. $|s-t| \leqq \frac{1}{2} T_{\ell-1}$, make identical contributions and cancel in $\delta \Pi$ [see (4.9)]. Therefore we may restrict the sums over $L$ by requiring that each $L$ contain at least one long line $s t \in L$ such that $|s-t| \geqq \frac{1}{2} T_{\ell-1}$. We write $\delta \Pi_{\ell}=\Pi_{\ell}^{\prime}-\Pi_{\ell-1}^{\prime}$, where the primes mean that the sums of laces, $L$, in Theorem 4.2 are constrained so that each lace contains at least one long bond $s t:|t-s| \geqq T_{\ell-1} / 2$. The method of bounding $\Pi$ applies also to $\delta \Pi^{\prime}$, after using $\left|\delta \Pi_{\ell}\right| \leqq\left|\Pi_{\ell}^{\prime}\right|+\left|\Pi_{\ell-1}^{\prime}\right|$. A long bond implies a long walk, so when we apply the arguments leading to (5.11) to 
each $\Pi^{\prime}$ we can assume that one $\tilde{C}$ norm is replaced by a norm on

$$
\delta \tilde{C}_{\ell}(x,|z|) \equiv \sum_{T_{\ell} \geqq s \geqq T_{\ell} / 6}|z|^{s} \tilde{C}_{\ell}(\cdot, s) .
$$

Define

$$
\left\|\delta^{*} \tilde{C}_{\ell}(\cdot,|z|)\right\|_{*}=\left\{\begin{array}{l}
\left\|\tilde{C}_{\ell}(\cdot,|z|)\right\|_{2} \\
\left\|\delta \tilde{C}_{\ell}(\cdot,|z|)\right\|_{\infty}
\end{array} \text { or } .\right.
$$

The bound is

$$
\left|\delta \Pi_{\ell}(k, z)\right| \leqq \frac{2}{1-\lambda} \sum_{i=1}^{\infty} \sum_{*} \prod_{j=1}^{2 i-1}\left(\left\|\delta^{*} \tilde{C}_{\ell}(\cdot,|z|)\right\|_{*}\right) \lambda^{i},
$$

where the sum over $*$ is a sum over all ways of allocating the $L_{\infty}$ norm. We have made use of the flexibility in $L_{\infty}$ norm in (5.8) to coordinate the $L_{\infty}$ norm with $\delta$.

All these arguments can be extended to derivatives with respect to $z$ and $k$ of $\delta \Pi$. We differentiate under the sum over laces, $L$, in Theorem 4.2. The $z$ derivatives act on the factor $z^{T}$ which we write as

$$
z^{T}=\prod_{j=1}^{2 i-1} z^{s_{i}}
$$

Each $z^{s}$ corresponds to a subwalk $\omega_{i}$ prescribed by lace $L$ We distribute the derivatives over the factors $z^{s}$ by Leibniz rule and proceed as before. $k$-derivatives act on the $\exp (i k \omega(T))$ in (4.8) which we can also factor along the walk $\omega=\omega_{1}$ $\cup \omega_{2} \ldots \cup \omega_{2 i-1}$, as prescribed by a lace $L$ Again we apply Leibniz rule, take absolute values inside the sum ways of distributing derivatives and proceed as above. We obtain a bound on $\delta \Pi$ in terms of $x$ space norms

\section{Proposition 5.2.}

$$
\left|\partial_{k}^{u} \partial_{z}^{v} \delta \Pi_{\ell}(k, z)\right| \leqq \frac{2}{1-\lambda} \sum_{i=1}^{\infty} \lambda^{i} \sum \prod_{j=1}^{2 i-1}\left\|(\cdot)^{u_{j}} \partial_{|z|}^{v_{j}} \delta^{*} \tilde{C}_{\ell}(\cdot,|z|)\right\|_{*},
$$

$v=0,1, \ldots,|u|=0,1,2,3, \ldots$, the unlabelled sum is over: non-negative multi-indices $u_{j}$ such that $\sum\left|u_{j}\right|=|u|$, non-negative $v_{j}$ 's such that $\sum v_{j}=v$ and choosing one \|\|$_{*}$ norm to be \|\|$_{\infty}$, the rest are \|\|$_{2}$ norms. In each $L_{2}$ norm $\delta^{*} \widetilde{C}_{\ell}=\widetilde{C}_{\ell}$. The $L_{\infty}$ norm has $\delta^{*} \widetilde{C}_{\ell}=\delta \widetilde{C}_{\ell}$ where

$$
\begin{aligned}
\tilde{C}_{\ell}(x,|z|) & \equiv \sum_{0 \leqq s \leqq T_{\ell}}|z|^{s} C_{\ell(s)}(x, s), \\
\delta \tilde{C}_{\ell}(x,|z|) & \equiv \sum_{T_{\ell} / 6 \leqq s \leqq T_{\ell}}|z|^{s} C_{\ell(s)}(x, s),
\end{aligned}
$$

and $\ell(s)$ is such that $T_{\ell(s)}=s$.

Note that the numbers of terms in the unlabelled sum is less than

$$
(2 i-1)^{1+|u|+v},
$$

which will be dominated by $\lambda$ powers if $\lambda$ is small. 
Now we use Proposition 5.2 to obtain Proposition 5.1, our main result for this section.

For $\ell \leqq m, T_{\ell} \geqq 1$,

$$
\begin{aligned}
\left\|\sum_{s \leqq T_{\ell}} \partial_{|z|}^{v}|z|^{s}(\cdot)^{u} \tilde{C}_{\ell(s)}(\cdot, s)\right\|_{2 \text { or } \infty} & \leqq \sum|z|^{s-v} s^{v}\left\|(\cdot)^{u} \widetilde{C}_{\ell(s)-1}(\cdot, s)\right\|_{2 \text { or } \infty}^{(x \text { space })} \\
& \leqq \sum|z|^{s-v} s^{v}\left\|\partial_{k}^{u} C_{\ell(s)-1}(\cdot, s)\right\|_{2 \text { or } 1}^{(k \text { space })}
\end{aligned}
$$

by the Hausdorff Young inequality, $u$ and $v$ are arbitrary. By the inductive assumption (I2), which we have for $\ell \leqq m-1$, and can use, because $\ell(s)-1 \leqq \ell-1$, $\leqq m-1$,

$$
\leqq K \sum_{s \leqq T_{\ell}}|z|^{s-v} s^{v} S^{|u| / 2-d /(2 p)} r_{\ell(s)-1}^{-s}
$$

with $p=2$ or 1 ,

$$
\leqq K T_{\ell}^{v+|u| / 2} \sum_{s \leqq T_{\ell}}\left(|z| / r_{\ell(s)-1}\right)^{s-v} S^{-d / 2 p},
$$

because $s \leqq T_{\ell}$ and for all $\ell, r_{\ell} \geqq 1$. The $|z|$ factor has to be bounded carefully, using (5.2)

$$
|z| / r_{\ell(s)-1} \leqq\left[r_{\ell-1}+2 \varepsilon_{\ell}\right] / r_{\ell(s)-1} \leqq 1+\left[r_{\ell-1}-r_{\ell(s)-1}\right]+2 \varepsilon_{\ell}
$$

By summing (I1) over $\ell$ from $\ell-1$ down to $\ell \in[\ell(s)-1, \ell(s)-2]$,

$$
\begin{aligned}
|z| / r_{\ell(s)-1} & \leqq 1+K \sqrt{\lambda} T_{\ell(s)-2}^{9 / 8-d / 2}+2 \varepsilon_{\ell} \\
& \leqq 1+K(\delta) \sqrt{\lambda} T_{\ell(s)}^{(9 / 8-d / 2) \delta}+2 \varepsilon_{\ell}
\end{aligned}
$$

for any $\delta<1$. Since $T_{\ell(s)}=s$ and $\delta$ can be chosen so that $(9 / 8-d / 2) \delta \leqq-1$,

$$
|z| / r_{\ell(s)-1} \leqq \exp \left[K(\delta) \sqrt{\lambda} s^{(9 / 8-d / 2) \delta}+2 \varepsilon_{\ell}\right] \leqq \exp \left(K(\delta) \sqrt{\lambda} s^{-1}+2 \varepsilon_{\ell}\right) .
$$

We substitute this upper bound for $|z| / r_{\ell(s)-1}$ into (5.16) to find that the norm (5.15) is less than

$$
K T_{\ell}^{v+u / 2} \sum_{s \leqq T_{\ell}} s^{-d / 2 p} \exp \left(2 s \varepsilon_{\ell}\right)
$$

By observing that the exponent is less than or equal to $\frac{1}{8} \log s$ for $s \geqq e$ we bound this by

$$
K T_{\ell}^{v+u / 2} \sum_{s} s^{-d / 2 p+\frac{1}{8}}
$$

Thus taking $p=2$ and $p=1$,

$$
\begin{gathered}
\left\|\sum_{s=0}^{T_{\ell}} \partial_{|z|}^{v}|z|^{s}(\cdot)^{u} \tilde{C}_{\ell(s)}(\cdot, s)\right\|_{2} \leqq K T_{\ell}^{v+u / 2}, \\
\left\|\sum_{T_{\ell} \geqq s \geqq \frac{1}{6} T_{\ell-1}} \partial_{|z|}^{v}|z|^{s}(\cdot)^{u} \tilde{C}_{\ell(s)}(\cdot, s)\right\|_{\infty} \leqq K T_{\ell}^{v+u / 2} T_{\ell-1}^{9 / 8^{-d / 2}} .
\end{gathered}
$$


We substitute these bounds into Proposition 5.2 and find, using $\sum v_{i}=v, \sum\left|u_{i}\right|=|u|$ that $\left|\partial_{k}^{u} \partial_{z}^{v} \delta \Pi_{\ell}(k, z)\right| \leqq \lambda K T_{\ell}^{v+u / 2} T_{\ell-1}^{9 / 8-d / 2}$ for $\ell \leqq m$, provided $\lambda$ is smaller than a universal constant. This is the desired bound (5.1), since $T_{\ell} \leqq K T_{\ell-1}^{a}$ for all $\ell$. The analyticity of $\delta \Pi$ in $z$ is immediate, the continuity in $k$ follows from the expansion (4.8) and the bounds we have proven. Proposition 5.1 is proved.

\section{Poles of $C_{\ell}(k, z)$}

In this section we shall obtain information about the poles of $C_{\ell}(k, z)$ using (5.1) and the inductive assumption I1. More precisely let

$$
\mathscr{D}_{\ell}(a)=\left\{z:|z| \leqq r_{\ell}+a \varepsilon_{\ell}\right\} .
$$

We shall prove that for $\ell \leqq m, C_{\ell}(k, z)$ is meromorphic in the disc $\mathscr{D}_{\ell}(3 / 2)$ and has a unique real pole $r_{\ell}(k)$ which varies differentiably with $k$ provided $k^{2} / 2 d \leqq \varepsilon_{\ell}$. These results will follow from implicit function type arguments.

Remark. By I1 note that

$$
0 \leqq r_{\ell}-r_{\ell-1} \leqq a\left(\varepsilon_{\ell-1}-\varepsilon_{\ell}\right) ; \quad \ell \leqq m-1,
$$

for $a \geqq 1 / 4$ and $\lambda$ smaller than a universal constant, hence the discs $\mathscr{D}_{\ell}(a)$ are decreasing in $\ell$, for $\ell \leqq m-1$.

Let us observe that $C_{\ell}(k, z)$ has the following symmetries:

i) $C_{\ell}(k, z)$ is even in $k_{i}, i=1, \ldots, d$,

ii) $C_{\ell}(k, \bar{z})=\overline{C_{\ell}(k, z)}$,

iii) $C_{\ell}(k+\pi, z)=C_{\ell}(k,-z)$,

where $\pi=(\pi, \ldots, \pi)$. Identities i) and ii) follow easily from using the invariance of the sum (3.4) under $\omega^{i} \rightarrow-\omega^{i}$, and iii) follows from the observation that $\omega^{1}(T)+\ldots+\omega^{d}(T), T$ are either both even or odd. The same symmetries hold with $C_{\ell}$ replaced by $\Pi_{\ell}$ (by definition of $\left.\Pi_{\ell}\right)$. From ii) it follows that the poles of $C_{\ell}(k, z)$ are real or come in complex conjugate pairs.

Lemma 6.1. Assume that I1 holds for $\ell \leqq m-1$ and (5.1) holds for $0 \leqq \ell \leqq m$. If $\lambda$ is taken small, independently of $m$, then

(a) I1 holds for $\ell \leqq m$,

(b) for $\ell \leqq m$, for all $k, \Pi_{\ell}(k, z)$ is analytic in $z, z \in \mathscr{D}_{\ell}(3 / 2)$ and

$$
\begin{aligned}
\left|\partial_{k}^{u} \partial_{z}^{v} \Pi_{\ell}(k, z)\right| & \leqq K(\delta) \lambda \quad \text { if } \quad d(u, v) \geqq \delta \\
& \leqq K \lambda\left(2+T_{\ell}\right)^{-d(u, v)} \text { if } d(u, v) \leqq 0
\end{aligned}
$$

for each $\delta>0, z \in \mathscr{D}_{\ell}(3 / 2)$,

(c) for $\ell \leqq m, C_{\ell}(k, z)$ is meromorphic in $z$ for $z \in \mathscr{D}_{\ell}(3 / 2)$.

In particular note that we have advanced one of our inductive hypotheses, I1. For the next lemma, recall that

$$
F_{\ell}(k, z) \equiv\left[1-z D(k)-\Pi_{\ell}(k, z)\right]^{-1} \equiv C_{\ell}^{-1}(k, z) .
$$

We will also need $r(k, \lambda=0)=D^{-1}(k)$, which is the unperturbed zero solving $1-r(k) D(k)=0$. 
Lemma 6.2. Assume the conclusions of Lemma 6.1. For $\lambda$ sufficiently small, independently of $m$, and all $\ell \leqq m$,

(a) When $k$ satisfies either $k^{2} \leqq 2 d \varepsilon_{\ell}$ or $(k-\pi)^{2} \leqq 2 d \varepsilon_{\ell}$, then $C_{t}(k, z)$ has one simple pole, $r_{t}(k)$, in $\mathscr{D}_{\ell}(3 / 2)$ whose derivatives $\partial_{k}^{u} r_{t}(k), 0 \leqq|u| \leqq 2$, are continuous, uniformly bounded and obey

$$
\partial_{k}^{u}\left(r_{\ell}(k)-r(k, \lambda=0)\right)=\lambda E .
$$

(b) With the same condition on $k$ as in part (a), $|u|, v \leqq 2$ :

$$
\int_{\partial \mathscr{D}_{\ell}(3 / 2)}\left|\partial_{k}^{u} C^{v+1}(k, z)\right||d z| \leqq K\left(2+T_{\ell}\right)^{|u|+v} \ln \left(2+\mathrm{T}_{\ell}\right) .
$$
and

(c) If the condition on $k$ in part (a) is violated, then $F_{t}(k, z)$ has no zero in $\mathscr{D}_{t}\left(\frac{1}{2}\right)$

$$
\int_{\partial \mathscr{D}_{\ell}(1 / 2)}\left|\partial_{k}^{u} C_{\ell}^{1+v}(k, z)\right||d z| \leqq K\left(2+T_{\ell}\right)^{|u|+v} \ln \left(2+T_{\ell}\right) .
$$

Proof of Lemma 6.1. First note that (6.2) holds for $\ell \leqq m-1$ by writing $\Pi_{\ell}$ as a telescopic sum of $\delta \Pi$ 's and bounding it with (5.1). We must check that the domains of $z$ are compatible, i.e., for $\ell^{\prime} \leqq \ell \leqq m-1$

$$
r_{\ell^{\prime}-1}+2 \varepsilon_{\ell^{\prime}} \geqq r_{\ell^{\prime}}+\frac{3}{2} \varepsilon_{\ell^{\prime}} \geqq r_{\ell-1}+\frac{3}{2} \varepsilon_{\ell-1}
$$

for $\ell \leqq m-1$. The first inequality follows from I1 and the second one follows from the remark after (6.1), provided $\lambda$ is taken smaller than a universal constant.

Part (a). To advance the induction step I1 we introduce an interpolating function: for $\ell \in(m-1, m), H(z, \sigma)=F_{\ell-1}(0, z)+\sigma \delta \Pi_{\ell}(0, z)$. Using (6.2) for $\ell \leqq m-1$ and (5.1) with $\ell \leqq m$, we see that

$$
\frac{\partial H}{\partial z}=-1+\lambda E \text { for }|z| \leqq r_{\ell-1}+3 / 2 \varepsilon_{\ell} .
$$

By taking $\lambda$ small (independently of $m$ ) and using the implicit function theorem $H(z, \sigma)$ has a locally unique simple zero $r(\sigma)$ as long as $\partial H / \partial z(r(\sigma), \sigma) \neq 0$. Local uniqueness forces $r(\sigma)$ to be real because zeros of $H$ are either real or occur in complex pairs.

Let $\bar{\sigma}$ be the largest value of $\sigma$ in $[0,1]$ such that $|r(\sigma)| \leqq r_{\ell-1}+\varepsilon_{\ell}$. For $\sigma \leqq \bar{\sigma}$ and $\lambda$ small

$$
\frac{d}{d \sigma} r(\sigma) \leqq\left|\frac{\frac{\partial H}{\partial \sigma}(r(\sigma), \sigma)}{\frac{\partial H}{\partial z}(r(\sigma), \sigma)}\right| \leqq\left|\delta \Pi_{\ell}(0, r(\sigma))\right| \cdot|(1+\lambda E)| \leqq K \lambda\left(2+T_{\ell-1}\right)^{-d / 2+9 / 8} \ll \varepsilon_{\ell},
$$

using (5.1). By integrating over $\sigma$ from 0 to $\bar{\sigma}$ we find that $\bar{\sigma}=1$ and

$$
r(\sigma=1)-r_{\ell-1} \leqq K \lambda\left(2+T_{\ell-1}\right)^{-d / 2+9 / 8} .
$$

Since $r_{\ell}$ lies on the positive real axis and is the zero of $F_{\ell}$ closest to the origin, local uniqueness forces $r(\sigma=1)=r_{\ell}$, hence I 1 holds for $\ell \leqq m$, provided we take $\lambda$ small so that $K \lambda \leqq K^{(11)} \sqrt{\lambda}$. 
Part (b). (6.2) is proved by writing $\Pi$ as a telescopic sum of $\delta \Pi$ 's and bounding each term using (5.1) for $\ell \leqq m$. By part (a) we now have

$$
r_{\ell^{\prime}-1}+2 \varepsilon_{\ell^{\prime}} \geqq r_{\ell}+\frac{3}{2} \varepsilon_{\ell} \quad \text { for } \quad \ell^{\prime} \leqq \ell \leqq m,
$$

so that the $z$ domains are compatible.

Part (c). Immediate consequence of part (b).

Proof of Lemma 6.2. Since the value of $\ell$ does not change in the course of this proof we suppress $\ell$ subscripts on $C_{\ell}, F_{\ell}, \Pi_{\ell}, T_{\ell}, \varepsilon_{\ell}, r_{\ell}$, and $\mathscr{D}_{\ell}(a)$.

From the definition of $F$ and (6.2),

$$
\frac{\partial F}{\partial z}=-D(k)-\frac{\partial \Pi}{\partial z}=-D(k)+\lambda E(k, z) .
$$

Suppose $k$ is near 0 or $\pi$ as in part (a) of the lemma, then $D(k)$ is bounded away from zero uniformly in $\ell$. Furthermore, if $F$ has two zeros $z(k)$ and $z^{\prime}(k)$ both in $\mathscr{D}(3 / 2)$, then the integral of $\partial F / \partial z$ along a contour between $z(k)$ and $z^{\prime}(k)$ must vanish. For $\lambda$ small this is in contradiction with (6.7). Thus for $\lambda$ small (uniformly in $\ell$ ) $F(z, k$ ) has at most one zero $r(k)$ in $\mathscr{D}(3 / 2)$ for each $k$ in the region specified. For small $k$, $r(k)$ is in $\mathscr{D}(3 / 2)$ and $r(k=0)=r$ by the implicit function theorem. Zeros are either real or occur in complex conjugates so by local uniqueness $r(k)$ lies on the real axis. Its $k$ derivatives are

$$
\frac{\partial r(k)}{\partial k_{i}}=-\frac{\partial F}{\partial k_{i}} / \frac{\partial F}{\partial z}=\left(\frac{1}{d} \sin \left(k_{i}\right) r(k)-\frac{\partial \Pi}{\partial k_{i}}(k, r(k))\right) / \frac{\partial F}{\partial z} .
$$

Since $\Pi$ is even in $k_{i},(6.2)$ implies

$$
\left|\frac{\partial \Pi}{\partial k_{i}}(k, r(k))\right| \leqq K \lambda\left|\sin k_{i}\right|
$$

for $k \simeq 0$ as in part (a). Thus using (6.2) again,

$$
\frac{\partial r(k)}{\partial k_{i}}=\frac{1}{d} \sin \left(k_{i}\right) r(k)(1+\lambda E(k, r(k))) / D(k) .
$$

We take $\lambda$ small (uniformly in $\ell$ ) and conclude that $r(k)$ moves monotonically away from $r(k=0)=r$ towards the boundary of $\mathscr{D}(3 / 2)$ along the real axis and (6.10) holds provided $r(k) \in \mathscr{D}(3 / 2)$ and $k^{2} \leqq 2 d \varepsilon$. By symmetry there is another trajectory of solutions starting at $-r$ when $k=\pi$ for which analogous considerations hold.

The second derivatives of $r(k)$ are obtained by twice differentiating $F(k, r(k))=0$ :

$$
0=\frac{\partial^{2} F}{\partial k_{i} \partial k_{j}}+\frac{\partial^{2} F}{\partial k_{i} \partial z} \frac{\partial r}{\partial k_{j}}+\frac{\partial^{2} F}{\partial k_{j} \partial z} \frac{\partial r}{\partial k_{i}}+\frac{\partial F}{\partial z} \frac{\partial^{2} r}{\partial k_{i} \partial k_{j}}+\frac{\partial^{2} F}{\partial z^{2}} \frac{\partial r}{\partial k_{i}} \frac{\partial r}{\partial k_{j}}
$$

By (6.10) and (6.2) every term in (6.11) except possibly the fourth is equal to its $\lambda=0$ value $+\lambda E$. For $k \cong 0$ or $k \cong \pi$ as in part (a) $\partial F / \partial z$ is bounded away from zero according to (6.2), so we obtain (6.3) with $|u|=2$ by solving (6.11) for the double derivative of $r$. At present $(6.3)$ is known provided $k^{2}$ or $(k-\pi)^{2} \leqq 2 d \varepsilon$ and 
$r(k) \in \mathscr{D}(3 / 2)$. By integrating (6.3) with $|u|=2$ from zero to $k$ using the fact that $\partial r / \partial k_{i}=0$ at $k=0$ (by symmetry) we find that $k^{2} \leqq 2 d \varepsilon \Rightarrow r(k) \in \mathscr{D}(3 / 2)$ provided $\lambda$ is smaller than a universal constant, consequently (6.3) is fully established for $|u|=2$. For $|u|=1$ it is immediate from (6.10). For $|u|=0$ we note that at $k=0$, $|r(k=0)-r(k=0, \lambda=0)| \leqq K \lambda$ by the implicit function theorem and (6.2), so we obtain (6.3) for $|u|=0$ by integrating (6.3) for $|u|=1$. Continuity of derivatives is evident.

Part (b). We will consider only the case $|u|=2, v=1$. The others are similar.

$$
\partial_{k}^{u} C(k, z)=2 C^{3}(k, z)\left(\partial_{k_{i}} F\right)\left(\partial_{k_{j}} F\right)-C^{2}(k, z) \partial_{k_{i}} \partial_{k_{j}} F .
$$

We bound derivatives of $F$ using (6.2). By integrating (6.3) with $|u|=2$ from zero to $k, k^{2} \leqq 2 d \varepsilon$, we find

$$
r(k)-r=r(k, \lambda=0)-1+\lambda E k^{2} .
$$

From this we conclude that if $\lambda$ is small (uniformly in $\ell$ ) and $k \cong 0$ or $k \cong \pi$ as required in parts (a) and (b) then $r(k)$ is strictly inside $\mathscr{D}(3 / 2)$, indeed

$$
|z-r(k)| \geqq \varepsilon / 4 \text { for } z \in \partial \mathscr{D}(3 / 2) .
$$

Therefore by the fundamental theorem of calculus

$$
|F(k, z)|=|z-r(k)| \mid \int_{0}^{1} d \sigma \partial_{z} F(k, r(k)+\sigma(z-r(k))|\geqq K| z-r(k) \mid,
$$

because by (6.7) $\partial_{z} F \simeq 1$, when $z \in \mathscr{D}(3 / 2)$ and $k \simeq 0$ or $\pi$. Thus by integrating the inverse of (6.15),

$$
\int_{\partial \mathscr{Q}(3 / 2)}|C(k, z)|^{n+1}|d z| \leqq(K \varepsilon)^{-n}|\ln K \varepsilon| .
$$

The logarithm is actually only necessary if $n=0$. We combine this inequality with (6.12) and obtain the inequality of part (b) for the case $|u|=2, v=1$.

Part (c). First let us suppose $2 d \varepsilon \leqq k^{2} \leqq 1$. To bound the contour integral we need a lower bound on $F$ :

$$
F(k, z)=F(0, z)+(1-D(k)) z-\int_{0}^{k} \partial_{k} \Pi(\tilde{k}, z) d \tilde{k}=\int_{r}^{z} \frac{\partial F}{\partial z}(0, \tilde{z}) d \tilde{z}+(1-D(k)) z+\lambda k^{2} E
$$

[using (6.9)]

$$
=r-D(k) z-\int_{r}^{z} \frac{\partial \Pi}{\partial z}(0, \tilde{z}) d \tilde{z}+\lambda k^{2} E=(r-D(k) z)+\lambda(z-r) E+\lambda k^{2} E
$$

[using (6.2)]. To prove that the error terms are relatively small we use $|a+i b|$ $\geqq|| a|+| b \mid / / \sqrt{2}$ to show that for $z \in \partial \mathscr{D}\left(\frac{1}{2}\right)$, i.e., $z=(r+\varepsilon / 2) e^{i \theta}$,

$$
|r-D(k) z| \geqq \frac{r}{\sqrt{2}}\left|1-D(k)\left(1+\frac{\varepsilon}{2 r}\right) \cos \theta\right|+\frac{1}{\sqrt{2}}|D(k)||\sin \theta| .
$$

For $2 d \varepsilon \leqq k^{2} \leqq 1, D(k)$ is bounded away from 1 in such a way that this inequality implies $|r-D(k) z| \geqq K\left(k^{2}+|D(k) \sin \theta|\right)$ with $K>0$. We now see that the last error 
term in (6.17) is relatively small when $\lambda$ is chosen small (uniformly in $\ell$ ). For the second term in (6.17) we use

$$
|r-z| \leqq|r-D(k) z|+|z||1-D(k)| \leqq|r-D(k) z|+\left(r+\frac{\varepsilon}{2}\right) \frac{1}{2 d} k^{2},
$$

and again we see that the first term dominates the second in (6.17). Thus for $\lambda$ small (uniformly in $\ell$ ) $|F(k, z)| \geqq K|r-D(k) z| \geqq K(\varepsilon+|\theta|$ ) for some $K$ 's $>0$. The bound (6.5) on the contour integral now follows with the same arguments as in (b). By symmetry the same arguments are available for $k$ such that $2 d \varepsilon \leqq(k-\pi)^{2} \leqq 1$. For the remaining values of $k,|1-z D(k)| \geqq K$, some $K>0$, and by (6.2) with $\lambda$ small $|C(k, z)| \leqq K$. Part (c) is proved.

\section{The Induction Completed. Proof of Main Theorems}

In this section we complete our induction by proving that $\mathrm{I} 2$ holds for $\ell \leqq m$, i.e.

$$
\left\|r_{\ell}^{T} \partial_{k}^{u} C_{\ell}(\cdot, T)\right\|_{L^{P}}^{(k \text { sace })} \leqq K^{(12)} T^{|u| / 2-d /(2 p)}
$$

for $T \geqq T_{\ell+1}$ and $\ell \leqq m$. We will prove (7.1) with $K^{(\text {I2) }}$ equal to 4 times (the best constant for $\lambda=0$ ).

We will also prove that there is a constant $D>0$ such that

$$
\left\langle\omega^{2}(T)\right\rangle_{\ell(T)}=-\sum_{i} \frac{\partial^{2}}{\partial k_{i}^{2}} C_{\ell(T)}(k, T) /\left.C_{\ell(T)}(0, T)\right|_{k=0}=D T\left(1+T^{-1 / 16} E\right)
$$

for $T$ large. Finally we shall establish the gaussian nature of the scaling limit of the end point, i.e. for fixed $t$

$$
\left\langle e^{i k \cdot \omega(s t) / \sqrt{s}}\right\rangle(\lambda) \equiv \frac{C_{\ell(s t)}\left(\frac{k}{\sqrt{s}},[s t]\right)}{C_{\ell(s t)}(0,[s t])} \Rightarrow e^{-D k^{2} t / 2},
$$

as $s$ tends to infinity.

Proof of 7.1. It suffices to establish (7.1) for $m-1 \leqq \ell \leqq m$. We may also assume that either $T$ or $T_{\ell}$ is large because we can achieve (7.1) on any bounded set of $T$ 's and $T_{\ell}$ 's by taking $\lambda$ small.

By Cauchy's formula

$$
C_{\ell}(k, T)=\frac{1}{2 \pi i} \oint C_{\ell}(k, z) z^{-T-1} d z=\frac{1}{2 \pi i} \oint \frac{z^{-T-1}}{F_{\ell}(k, z)} d z,
$$

where the contour is a small circle centered at the origin. If $k^{2} \geqq 2 d \varepsilon_{\ell}$ and $(k-\pi)^{2}$ $\geqq 2 d \varepsilon_{\ell}$, then by Lemma $6.2, C_{\ell}(k, z)$ is analytic inside the disc $\mathscr{D}\left(\frac{1}{2}\right)$. We now deform the contour in (7.4) to $\partial \mathscr{D}_{\ell}\left(\frac{1}{2}\right)$. By (6.5) and (7.4) we see that

$$
\begin{aligned}
r_{\ell}^{T}\left|\partial_{k}^{u} C_{\ell}(k, T)\right| & <\sup _{z \in \partial \mathscr{D}_{\ell}(1 / 2)} K T_{\ell}^{|u|} \ln T_{\ell}\left|r_{\ell} / z\right|^{T} \\
& =K T_{\ell}^{|u|} \ln T_{\ell}\left|r_{\ell} /\left(r_{\ell}+\varepsilon_{\ell} / 2\right)\right|^{T} \\
& \leqq K T_{\ell}^{|u|} \ln T_{\ell} e^{-T_{\ell} / 4} \leqq \frac{1}{4} K^{(12)} T^{-d / 2}(2 \pi)^{-d}
\end{aligned}
$$

for $T \geqq T_{\ell+1}$ and either $T$ or $T_{\ell}$ large. 
For the case $k^{2} \leqq 2 d \varepsilon_{\ell}$ we deform the small contour about the origin to $\mathscr{C}=\partial \mathscr{D}_{\ell}(3 / 2)$, which by Lemma 6.2 contains exactly one pole $r_{t}(k)$. Thus we have a contribution from the pole plus an error term:

$$
r_{\ell}^{T} C_{\ell}(k, T)=-\left[\frac{\partial F_{\ell}}{\partial z}\left(k, r_{\ell}(k)\right)\right]^{-1} r_{\ell}(k)^{-T-1} r_{\ell}^{T}+r_{\ell}^{T} \oint_{\mathscr{\zeta}} C_{\ell}(k, z) z^{-(T+1)} d z .
$$

As in our analysis of (7.5) we apply (6.4) to show that the second term on the right side of (7.6) is bounded by $\frac{1}{4} K^{(12)} T^{-d / 2}$ for $T \geqq T_{\ell+1}$. To bound the first term on the right side of (7.6) we integrate (6.3) with $|u|=2$ twice with respect to $k$, remembering that at $k=0$ first derivatives vanish so that

Thus

$$
r_{\ell}(k)-r_{\ell}=r(k, \lambda=0)-1+\lambda k^{2} E=D^{-1}(k)-1+\lambda k^{2} E .
$$

$$
\begin{aligned}
& \int_{k^{2} \leqq 2 d \varepsilon_{\ell}} d k\left|\left(\partial_{z} F_{\ell}\right)^{-1} r_{\ell}(k)^{-T-1} r_{\ell}^{T}\right|^{p} \\
& \sim\left(\partial_{z} F_{\ell}\right)^{-1}(k=0) r_{\ell}^{-1} \int_{-\infty}^{\infty} d k \exp \left(-\frac{p}{2} K(\ell, \lambda) T k^{2}\right),
\end{aligned}
$$

where $\sim$ means asymptotic as $T \rightarrow \infty$, uniformly in $\ell$ and $K(\lambda, \ell) \rightarrow 1$ uniformly in $\ell$ as $\lambda \rightarrow 0$. From this we immediately obtain (7.1) for $u=0$ provided $\lambda$ is taken smaller than a universal constant.

Next we consider the case $|u|=2, k^{2} \leqq 2 d \varepsilon_{\ell}$. We take the second derivative of both sides of (7.6). The second term on the right is again bounded by $T^{-d / 2}$ using (6.4) as before. To analyze the first term let us set $r=r_{\ell}(k)$ and

$$
H=\left[\frac{\partial F_{\ell}}{\partial z}\left(k, r_{\ell}(k)\right)\right]^{-1} \text {. }
$$

Then we have

$$
\begin{gathered}
\frac{d^{2}}{d k_{i}^{2}}\left[H r^{-(T+1)}\right]=\left\{\frac{d^{2}}{d k_{i}^{2}} H\right\} r^{-(T+1)}-2\left(\frac{d}{d k_{i}} H\right)(T+1) r^{-(T+2)} \frac{\partial r}{\partial k_{i}} \\
+H\left(\frac{\partial}{\partial k_{i}} r\right)^{2}(T+1)(T+2) r^{-(T+3)}-H\left(\frac{d^{2}}{d k_{i}^{2}} r\right)(T+1) r^{-(T+2)}
\end{gathered}
$$

After multiplying by $r_{t}(0)^{T}$ we shall show that the last two terms of (7.7) are dominant in $L^{p}$ norm for large $T$. Moreover if $k=0$, the second and third terms on the right side of (7.7) vanish because $r_{t}(k)$ is even. To show that the first two terms are relatively small we shall use the following lemma.

Lemma 7.1. For $k^{2} \leqq 2 d \varepsilon_{\ell}$ we have

$$
\begin{gathered}
\left|\frac{d}{d k_{1}} H\right|<K T^{\beta}\left(1+T^{1 / 2}\left|k_{1}\right|\right), \\
\left|\frac{d^{2}}{d k_{1}^{2}} H\right|<K\left[T^{2 \beta}\left(1+T^{1 / 2}\left|k_{1}\right|\right)^{2}+T^{1 / 2+\beta}+T^{1+\beta}\left|k_{1}\right|+T^{3 / 2+\beta} k_{1}^{2}+T^{1 / 2+\beta}\right],
\end{gathered}
$$

where $\beta=\frac{1}{8}+2(\alpha-1)$. 
Proof. The total derivatives with $\ell$ subscripts suppressed are given by

$$
\frac{d}{d k_{1}} H=-H^{2}\left[\frac{\partial^{2} F}{\partial k_{1} \partial z}+\frac{\partial^{2} F}{\partial z^{2}} \frac{\partial r}{\partial k_{1}}\right]
$$

and

$$
\begin{aligned}
\frac{d^{2}}{d k_{1}^{2}} H= & 2 H^{3}\left[\frac{\partial^{2} F}{\partial k_{1} \partial z}+\frac{\partial^{2} F}{\partial z^{2}} \frac{\partial r}{\partial k_{1}}\right]^{2} \\
& -H^{2}\left[\frac{\partial^{3}}{\partial^{2} k_{1} \partial z} F+2 \frac{\partial^{3} F}{\partial k \partial z^{2}} \frac{\partial r}{\partial k}+\frac{\partial^{3} F}{\partial z^{3}}\left(\frac{\partial r}{\partial k_{1}}\right)^{2}+\frac{\partial^{2} F}{\partial z^{2}} \frac{\partial^{2} r(k)}{\partial^{2} k_{1}}\right]
\end{aligned}
$$

The lemma now follows directly by applying (6.2) and (6.3) to each of the terms above together with the bound $\left|\partial r / \partial k_{1}\right| \leqq K\left|k_{1}\right|$ (see 6.10). We multiply (7.7) by $r_{\ell}^{T}(0)$ and take the $L^{P}$ norm of both sides remembering $k^{2} \leqq 2 d \varepsilon_{\ell}$. We analyse $\left[r_{\ell}(k) / r_{\ell}\right]^{T}$ as in the $u=0$ case, and we use Lemma 6.2 part (a) on $k$ derivatives of $r_{\ell}(k)$. The above lemma shows that the first two terms of (7.7) give contributions which are down by $T^{1 / 8}$ relative to the second two terms, hence they can be dropped. The rest of the argument is as in the $|u|=0$ case. Mixed partials and the case $|u|=1$ are analogous. End of proof of (7.1).

Proof of (7.2). We begin by showing that for $k^{2} \leqq K d \varepsilon_{\ell}$, with $K$ sufficiently small,

$$
C_{\ell}(k, T)=-\left[\frac{\partial F}{\partial z}\left(k, r_{\ell}(k)\right)\right]^{-1} r_{\ell}^{-T-1}(k)\left[1+T^{-1 / 16} E\right]
$$

for $T \leqq T_{\ell}$. Here $\ell \equiv \ell(T)$ is defined so that $T_{\ell}=T$. If $\ell=\ell(T)=\ell$, then recall that $C_{\ell}(k, T)=C_{\ell_{1}}(k, T)$. By (7.6)

$$
C_{\ell}(k, T)=-\left[\frac{\partial F_{\ell}}{\partial z}\left(k, r_{\ell}(k)\right]^{-1} r_{\ell}(k)^{-T-1}+\frac{1}{2 \pi i} \oint C_{\ell}(k, z) z^{-T-1} d z,\right.
$$

where $\mathscr{C} \equiv \partial \mathscr{D}_{\ell}(3 / 2)$ is the integration contour. By integrating with respect to $k(6.3)$ with $|u|=2$, we find that $r_{\ell}(k)=r_{\ell} D^{-1}(k)+\lambda E k^{2}$, so that for $\lambda$ small $r_{\ell}(k) \in \mathscr{D}_{\ell}(1 / 16)$. For $z \in \mathscr{C}$ we have

$$
\left|r_{\ell}(k) / z\right|^{T+1} \leqq\left[\left(r_{\ell}+\frac{1}{16} \varepsilon_{\ell}\right) /\left(r_{\ell}+\frac{3}{2} \varepsilon_{\ell}\right)\right]^{T+1} \leqq K T^{-5 / 64} .
$$

By (6.7), (6.4) and the above estimate the final term of (7.9) is bounded and (7.8) follows.

The second derivative of $C(k, T)=C_{\ell(T)}(k, T)$ at $k=0$ is again calculated using (7.6) with $\mathscr{C}$ as above. By (7.6), (7.7), and Lemma 7.1, we have

$$
\begin{aligned}
\left.r^{T+1}(0) \frac{\partial^{2} C(k, T)}{\partial^{2} k_{i}}\right|_{k=0}= & {\left[\frac{\partial F}{\partial z}(0, r(0))\right]^{-1} \frac{\partial^{2} r}{\partial k_{i}^{2}}(T+1) \frac{1}{r(0)}+T^{(1 / 2+\beta)} E } \\
& +\left.\frac{1}{2 \pi i} \oint_{\mathscr{c}} C^{2}(k, z) \frac{\partial^{2} F}{\partial k_{i}^{2}}\left(\frac{r}{z}\right)^{T+1} d z\right|_{k=0} . \cdot
\end{aligned}
$$

For notational simplicity we have set $r=r_{\ell(T)}(k), F_{\ell(T)}=F$ etc. The term $T^{(1 / 2+\beta)} E$ represents contributions arising from $d^{2} H /\left.d k_{i}^{2}\right|_{k=0}$. Note the $\partial H / \partial k$ and $\partial r / \partial k$ 
vanish at $k=0$. The final term on the right side of (7.10) is bounded by $K T^{15 / 16}$, using (6.4) and the fact that for $z \in \mathscr{C}|r(0) / z|^{T} \leqq K T^{-5 / 64}$. Thus (7.8) and (7.10) imply that

$$
-\frac{\partial^{2}}{\partial k_{i}^{2}} C(k, T) /\left.C(0, T)\right|_{k=0}=\left.\frac{1}{r}\left(\frac{\partial^{2}}{\partial k_{i}^{2}} r\right)\right|_{k=0} T+T^{15 / 16} E .
$$

To complete our proof of (7.2) we need only show that the $T$ dependence of $r=r_{\ell(T)}$ and its second derivative in $k$ are of lower order so that the diffusion constant $D$ in (7.2) may be chosen independent of $T$. First note that by $\mathrm{I} 1,\left|r_{\infty}-r_{\ell(T)}\right| \leqq K T^{-5 / 4}$. We need to obtain a similar bound for the second derivative of $r_{\ell(T)}(k)$. Let $\ell=\ell(T)$. By (6.11) and (6.2),

$$
\left.\frac{\partial^{2} r_{\ell}}{\partial k_{i}^{2}}(k)\right|_{k=0}=-\left.\frac{\partial^{2} F_{\ell}}{\partial k_{i}^{2}}\left(k, r_{\ell}(0)\right)\left(\frac{\partial F}{\partial z}\left(0, r_{\ell}\right)\right)^{-1}\right|_{k=0} .
$$

The $\ell$ dependence of the first factor on the right side of (7.11) can be analyzed as follows:

$$
\begin{aligned}
\frac{\partial^{2} F_{\ell}}{\partial k_{i}^{2}}\left(k, r_{\ell}(0)\right)-\left.\frac{\partial^{2} F_{\ell}}{\partial k_{i}^{2}}\left(k, r_{\infty}\right)\right|_{k=0} & \leqq \sup _{z \in \mathscr{D}_{\ell}(1 / 2)}\left|\frac{\partial^{3}}{\partial z \partial k_{i}^{2}} F_{\ell}(k, z)\right|_{k=0}\left|r_{\ell}-r_{\infty}\right| \\
& \leqq K T_{\ell}^{1 / 2+\beta} T_{\ell}^{-5 / 4} \leqq K T_{\ell}^{-3 / 4+\beta} \leqq K T_{\ell}^{-1 / 2}
\end{aligned}
$$

Recall that $r_{\infty}(0)$ belongs to $\mathscr{D}_{t}\left(\frac{1}{2}\right)$. Also

$$
\begin{aligned}
\sum_{\ell_{1} \leqq} \frac{\partial^{2}}{\partial k_{i}^{2}}\left(F_{\ell_{1}}\left(k, r_{\infty}(0)\right)-F_{\ell_{1}+1}\left(k, r_{\infty}(0)\right)\right) & =\sum_{\ell_{1} \geqq \ell} \frac{\partial^{2}}{\partial k_{i}^{2}} \Pi_{\ell_{1}+1}=\sum_{\ell_{1} \geqq \ell} T_{\ell_{1}+1}^{-1 / 4} E \\
& =T_{\ell}^{-1 / 4} E .
\end{aligned}
$$

Hence $\partial_{k}^{2} F_{\ell}$ may be replaced by $\partial_{k}^{2} F_{\infty}$. The second factor of (7.11) may be analysed in a similar fashion, hence (7.2) follows.

Proof of the Scaling Limit (7.3). Let $s t=T$. For any given values of $k$ and $t$ and $K>0$ we have $k^{2} / s \leqq K d \varepsilon_{\ell(T)}$ as $s \rightarrow \infty$. Thus by (7.8) with $\ell=\ell(T)$ we have

$$
C_{\ell}\left(\frac{k}{\sqrt{s}}, T\right)=-\left[\frac{\partial F_{\ell}}{\partial z}\left(\frac{k}{\sqrt{s}}, r_{\ell}(k / \sqrt{s})\right)\right]^{-1} r_{\ell}\left(\frac{k}{\sqrt{s}}\right)^{-T-1}\left(1+T^{-1 / 16} E\right) .
$$

Lemma 7.1 implies that

$$
\left|\frac{d}{d k_{i}}\left[\frac{\partial F_{\ell}}{\partial z}\left(\frac{k}{\sqrt{s}}, r_{\ell}(k / \sqrt{s})\right)\right]^{-1}\right| \leqq K(t) T^{-1 / 8},
$$

so we can conclude that $C_{\ell}(k / \sqrt{s}, T) \cong C_{\ell}(0, T)$ in that

$$
\lim _{s \rightarrow \infty} C_{\ell(T)}(k / \sqrt{s}, T) / C_{\ell(T)}(0, T)=\lim _{s \rightarrow \infty}\left[\frac{r_{\ell(T)}(0)}{r_{\ell(T)}(k / \sqrt{s})}\right]^{T} .
$$

By Taylor's theorem with remainder

$$
r_{\ell(T)}(k / \sqrt{s})=r_{\ell(T)}(0)+\sum_{|u|=2} \partial^{u} r_{\ell(T)}(\xi) \frac{k^{u}}{s u !},
$$


where $\xi$ lies on the line segment joining 0 to $k / \sqrt{s}$. Note that $\partial^{u} r_{\ell(T)}(0)$ vanishes for $|u|=1$ and for mixed partials with $|u|=2$. The proof of the scaling limit follows once we show that the following limit exists for all $k$ and $t$

$$
\lim _{s \rightarrow \infty} \frac{1}{r_{\ell(T)}} \partial^{u} r_{\ell(T)}(\xi)=D^{u}, \xi \in\{\sigma k / \sqrt{s}: 0 \leqq \sigma \leqq 1\},
$$

where $D^{u}$ is a constant independent of $k$ and $t$, which vanishes for mixed partials. Note that $D^{i i}=D$, the diffusion constant.

To establish the limit we return to (6.11) evaluated at $k=\xi$. The second, third and fifth terms in (6.11) go to zero as $s t \equiv T \rightarrow \infty$ by (6.2) and (6.3) with $|u|=2$ integrated from $k=0$ to $\xi$. Existence of $D^{u}$ follows as soon as we establish limits for the coefficient $\partial F / \partial z$ and the first term in (6.11).

Since $\partial^{u} \delta \Pi_{j}(k, z)$ is continuous in $k$ for $|u| \leqq 2$ and is exponentially small for large $j$, it follows by summing over $j$ that

$$
\lim _{s \rightarrow \infty}\left|\partial^{u} F_{\ell}\left[\xi(s), r_{\ell}(\xi(s))\right]-\partial^{u} F_{\ell}\left[0, r_{\ell}(\xi(s))\right]\right|=0 .
$$

As in (7.12) one can show that

$$
\mid \partial^{u} F_{\ell}\left(0, r_{\ell}(\xi(s))-\partial^{u} F_{\ell}\left(0, r_{\infty}(0)\right) \mid \Rightarrow 0 .\right.
$$

Finally $\partial^{u} F_{\ell}\left(0, r_{\infty}(0)\right)$ is Cauchy by (5.1) together with the fact that $r_{\infty}(0) \in \mathscr{D}_{\ell}\left(\frac{1}{2}\right)$ for all $\ell$. The argument for $\partial F / \partial z$ in (6.11) is similar.

Acknowledgements. The authors would like to thank Mark Kac, Jürg Fröhlich, Alan Sokal, and Eugene Dynkin for teaching us about this and related problems.

\section{References}

1. Domb, C.: Self-avoiding random walk on lattices. In: Stochastic processes in chemical physics. Shuler, K. E. (ed.), pp. 229-260. New York: Wiley 1969

2. Aizenman, M.: Geometric analysis of $\phi^{4}$ fields and Ising models. Parts I and II. Commun. Math. Phys. 86, 1-48 (1982)

3. Fröhlich, J.: On the triviality of $\lambda \phi_{d}^{4}$ theories and the approach to the critical point in $d \geqq 4$ dimensions. Nucl. Phys. B 200, 281-296 (1982)

4. Lawler, G.: A self-avoiding random walk. Duke Math. J. 47, 655-693 (1980)

5. Fröhlich, J. (ed.): Scaling and self-similarity in physics - Renormalization in statistical mechanics and dynamics. Boston, Basel, Stuttgart: Birkhäuser 1983

6. Fröhlich, J.: Cargèse lectures

Communicated by A. Jaffe

Received April 2, 1984; in revised form June 14, 1984 